\title{
Tissue-specific expression and subcellular localization of ALADIN, the absence of which causes human triple A syndrome
}

\author{
A-Ri Cho ${ }^{1,3 *}$, Keum-Jin Yang ${ }^{1,3 *}$, Yoonsun Bae ${ }^{1,3}$, \\ Young Yil Bahk ${ }^{2}$, Eunmin Kim ${ }^{1,3}$, Hyungnam Lee ${ }^{1}$, \\ Jeong Ki Kim ${ }^{1,3}$, Wonsang Park ${ }^{4}$, \\ Hyanshuk Rhim ${ }^{1,3}$, Soo Young Choi $^{5}$, \\ Tsuneo Imanaka ${ }^{6}$, Sungdae Moon ${ }^{7}$, \\ Jongbok Yoon ${ }^{2}$ and Sungjoo Kim Yoon ${ }^{1,3,8}$ \\ ${ }^{1}$ Research Institute of Molecular Genetics \\ The Catholic University of Korea \\ Seoul 137-040, Korea \\ ${ }^{2}$ Department of Biochemistry, PNRC \\ Yonsei University \\ Seoul 120-752, Korea \\ ${ }^{3}$ Department of Biomedical Sciences \\ ${ }^{4}$ Department of Pathology \\ The Catholic University of Korea \\ Seoul 137-040, Korea \\ ${ }^{5}$ Division of Life Sciences \\ College of Natural Sciences, Hallym University \\ Chuncheon 200-702, Korea \\ ${ }^{6}$ Graduate School of Medicine and Pharmaceutical Sciences \\ University of Toyama, 2630 Sugitani \\ Toyama 940-0194, Japan \\ ${ }^{7}$ Department of Internal Medicine \\ The Catholic University of Korea \\ Seoul 137-040, Korea \\ ${ }^{8}$ Corresponding author: Tel, 82-2-2258-7474; \\ Fax, 82-2-594-2385; E-mail, sjkyoon@ catholic.ac.kr \\ *These authors contributed equally to this work. \\ DOI 10.3858/emm.2009.41.6.043
}

Accepted 6 January 2009

Abbreviations: $A A A S$, achalasia-addisonianism-alacrima syndrome gene; ALADIN, alacrima-achalasia-adrenal insufficiency neurologic disorder; EEA1, early endosome antigen 1; ORF, open reading frame; PARP, poly ADP-ribose polymerase; WD, tryptophan aspartic acid

\footnotetext{
Abstract

Triple A syndrome is a rare genetic disorder caused by mutations in the achalasia-addisonianism-alacrima syndrome (AAAS) gene which encodes a tryptophan aspartic acid (WD) repeat-containing protein named alacrima-achalasia-adrenal insufficiency neurologic disorder (ALADIN). Northern blot analysis shows that
}

the $2.1 \mathrm{~kb} A A A S$ mRNA is expressed in various tissues with stronger expression in testis and pancreas. We show that human ALADIN is a protein with an apparent molecular weight of $60 \mathrm{kDa}$, and expressed in the adrenal gland, pituitary gland and pancreas. Furthermore, biochemical analysis using anti-ALADIN antibody supports the previous finding of the localization of ALADIN in the nuclear membrane. The mutations S544G and S544X show that alteration of S544 residue affects correct targeting of ALADIN to the nuclear membrane.

Keywords: AAAS protein, human; gene expression profiling; protein transport

\section{Introduction}

Triple A syndrome, also known as Allgrove syndrome (AS, OMIM 231550), is a rare human autosomal recessive disorder. The phenotype of the triple A syndrome features adrenocorticotropic hormone (ACTH)-resistant adrenal failure, achalasia of the cardia and alacrima (Moore et al., 1991). In search of the gene responsible for AS, the triple A syndrome locus was linked to the chromosome $12 q 13$ region (Weber et al., 1996), and positional cloning efforts led to isolation of the AS-causing gene, achalasia-addisonianism-alacrima syndrome gene (AAAS) (Tallio-Pelet et al., 2000). The AAAS gene encodes a 546-amino acid (aa) protein named alacrima-achalasia-adrenal insufficiency neurologic disorder (ALADIN), a new member of the family of tryptophan aspartic acid (WD) repeat- containing proteins.

Currently, all reported AS patients harbour mutations in the AAAS gene. Thirteen nonsense mutations, ten frameshift and five aberrant splicing mutations have been described in patients with AS (Brooks et al., 2005). All these mutations are predicted to produce truncated proteins lacking the C-terminus, thus suggesting the importance of this region for effective ALADIN function. Five missense mutations, four in WD domains and one in the 15th aa, have also been reported. Recently, ALADIN was reported as localizing to the nuclear pore complex (NPC), and the mutants with a variety of disease-associated missense, nonsense, and frameshift mutations failed to localize to NPCs and were found predominantly in the cytoplasm. But 
Q15K localized to NPCs, suggesting that this residue may be critical for the interaction of ALADIN with a protein(s) essential for the function of ALADIN but not involved in NPC localization (Cronshaw and Matunis, 2003).

$A A A S$ is ubiquitously expressed in all tissues tested (Tullio-Pelet et al., 2000), but the expression of ALADIN is not reported until now.

In the present study, we determined the tissue specific expression pattern of $A A A S$ gene in mRNA level using multiple northern blot and protein level using antibodies raised against ALADIN. These analysis may be useful in understanding of tissuespecific symptoms of AS. In addition, we further defined the minimal requirement for ALADIN targeting to the NPC using artificial mutant constructs with altered C-termini.

\section{Results and Discussion}

\section{Cloning of a full-length $A A A S \mathrm{CDNA}$}

We identified a $1.4 \mathrm{~kb}$ insert clone, 282D10, from the normalized infant brain cDNA library (Soares et al.,1994) containing the EST 1190E. Because the mRNA transcripts for the EST 1190E were longer than $1.4 \mathrm{~kb}$, the full-length cDNA was cloned by performing 5' RACE experiments using human liver total RNA as a template. The analysis of the full-length cDNA sequence revealed that it was identical to that of the AAAS gene (NM_015665).

\section{Tissue distribution of AAAS mRNA in human}

We performed Northern blot analysis to determine the tissue specificity of $A A A S$ expression and the size of the transcript. The C-terminal probe spanning exons 4-16 recognized two transcripts, 2.1 and $2.7 \mathrm{~kb}$ in sizes (Figure 1). All tissues expressed both transcripts at various expression levels. A $85 \mathrm{bp}$ DNA fragment representing a part of the first exon specifically recognized the $2.1 \mathrm{~kb}$ mRNA only. This exon contains the start codon of the AAAS gene, thus indicating that the $2.1 \mathrm{~kb}$ mRNA is the transcript encoding ALADIN. Currently, we know neither the nature of the $5^{\prime}$ end of the $2.7 \mathrm{~kb}$ transcript nor the protein this transcript might encode and can not rule out the possibility that two transcripts are produced by alternative splicing in AAAS gene. The $2.1 \mathrm{~kb}$ mRNA was widely expressed in human tissues with strong expression in testis, pancreas, kidney and placenta (Figure 1).

The relative tissue expression pattern of $A A A S$ mRNA reported previously comprises data from tissues expressing both 2.1 and $2.7 \mathrm{~kb}$ transcripts, as the pattern was obtained by dot blot analysis using a probe spanning exons 7-14 (Tullio et al., 2000). In fact, the MTN blots probed with the DNA fragment corresponding to exons 7-14 displayed a tissue expression pattern identical to that seen with the probe encompassing exons 4-6 (Supplemental Data Figure S1 and Figure 1). Recently, it has been reported that the splice variant of human AAAS, AAAS-v2, which contains three WD40 domains without exon 6 (Li et al., 2005). We do not know yet whether this variant is identical to our splice variant since we did not characterize the 2.7 $\mathrm{kb}$ transcript. But we think that $A A A S-\mathrm{v} 2$ is a different transcript from 2.1 and $2.7 \mathrm{~kb}$ transcripts in our report because these were expressed in heart, placenta, spleen, prostate, testis, colon and

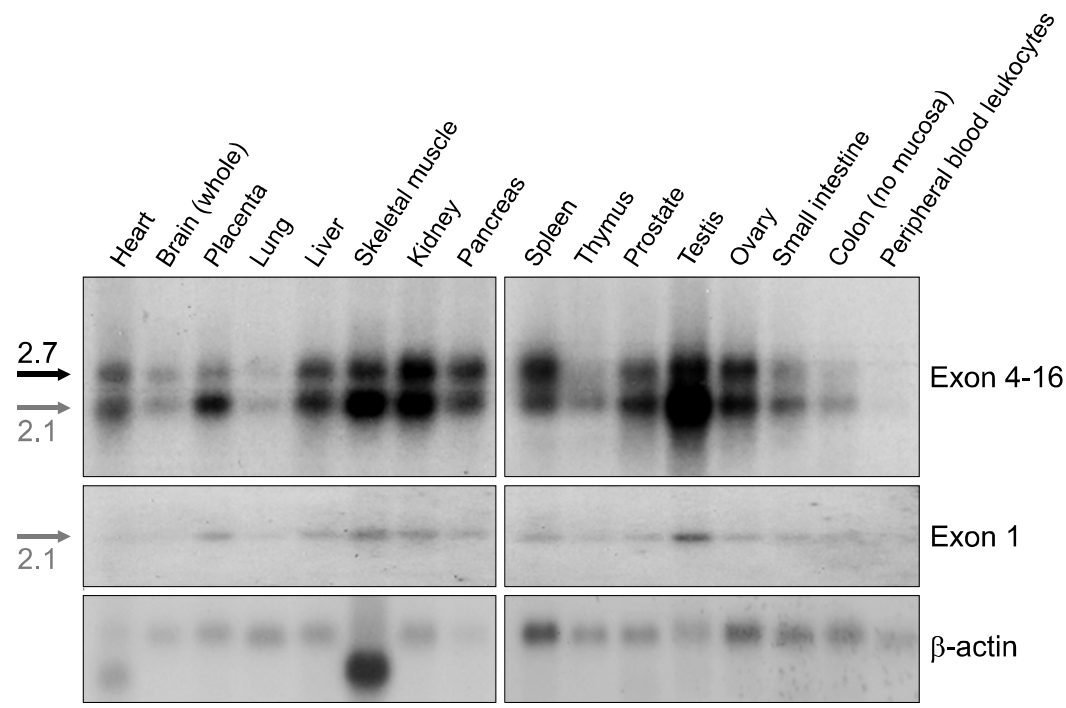

Figure 1. Expression of $A A A S \mathrm{mRNA}$ in human tissues. To determine the tissue distribution of human AAAS mRNA, the MTN blots were probed with either exon 4-16 or a part of exon 1. 
A

1

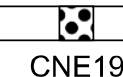

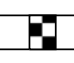

CVL16
B

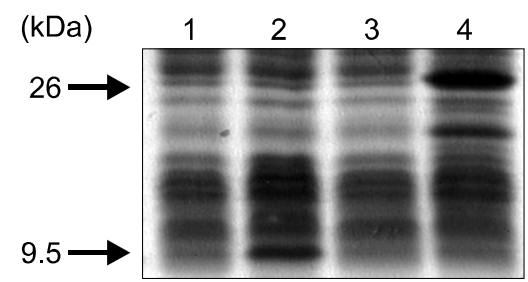

C
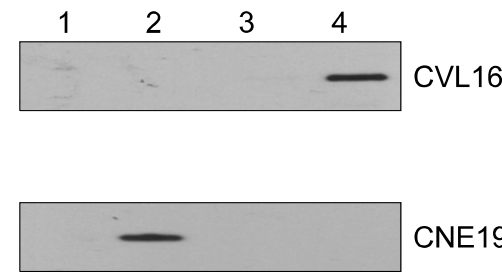

CNE19
D

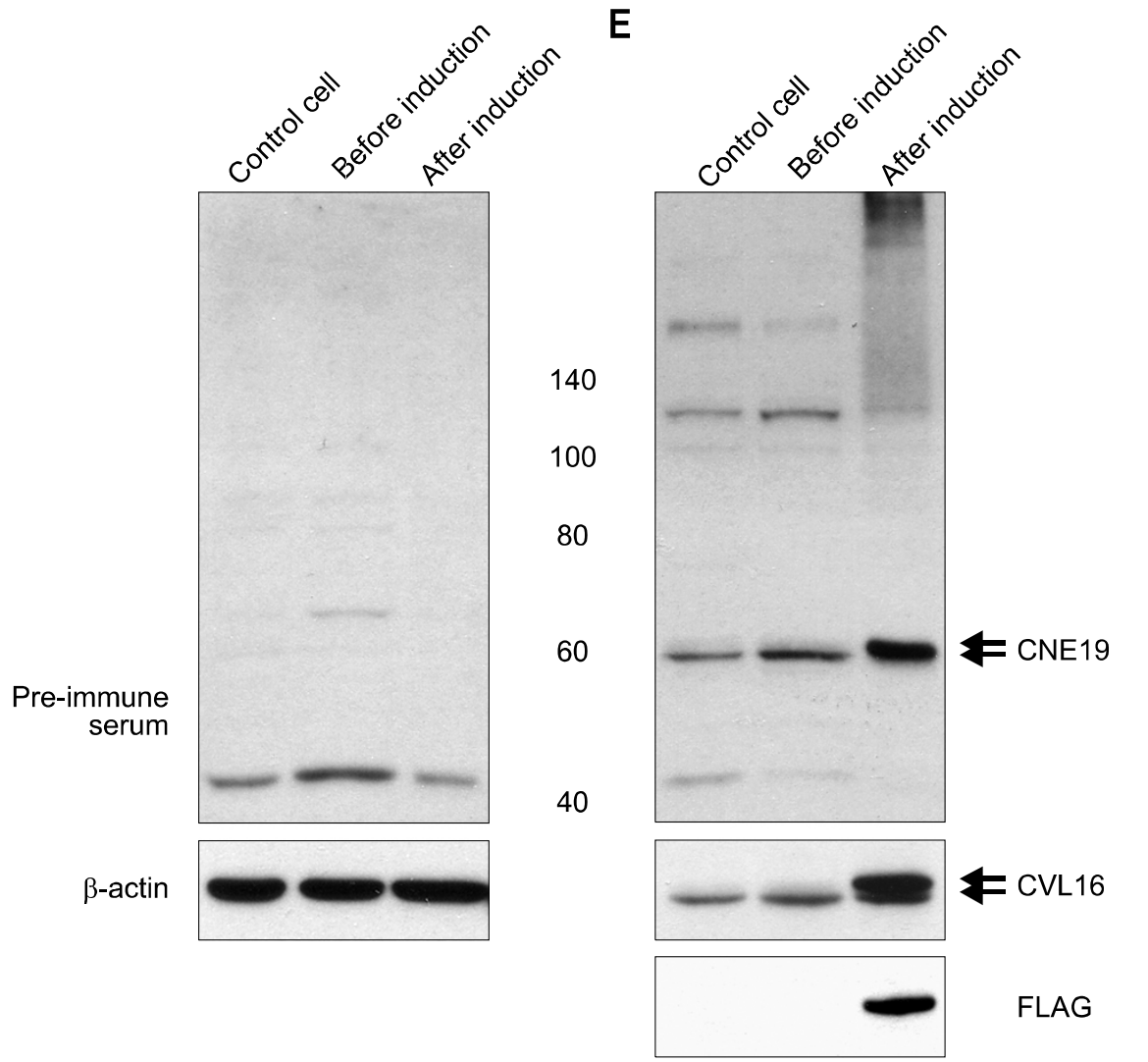

Figure 2. ALADIN-specific peptide antibodies. (A) Antigen sequences of ALADIN. The dark grey box represents the WD repeat domain. (B) Protein extracts from Escherichia coli cells expressing a His-tagged N-terminal 33 aa ALADIN sequence (lanes 1 and 2) and a His-tagged C-terminal 166 aa ALADIN sequence (lanes 3 and 4 ) were separated on $15 \%$ (w/v) SDS-PAGE. Lanes 1, 3: lysates prior to induction; 2, 4: lysates after induction. Proteins were stained with Coomassie Brilliant Blue. Arrows indicate the induced His-tagged proteins. (C) The CNE19 antibody recognized a $9.6 \mathrm{kDa}$ protein, while the CVL16 antibody recognized a $26 \mathrm{kDa}$ protein. (D) and (E) Western blot analysis of ALADIN in HeLa cells. FLAG-tagged ALADIN was expressed using Tet-off system as described previously (Min et al., 2003). Upper and lower arrows indicate FLAG-ALADIN and endogenous ALADIN, respectively. peripheral blood leukocyte, while $A A A S$-v2 was not expressed in these tissues and had a $1.7 \mathrm{~kb}$ fulllength cDNA.

\section{Expression of ALADIN in human tissues}

The expression of the AAAS gene product, ALADIN, was investigated by western blot analysis using antibodies raised against two separate peptides (Figure 2A-C). While anti-FLAG antibodies detected only fusion proteins, antibodies CNE19 and CVL16 detected an additional protein of $60 \mathrm{kDa}$ from $\mathrm{HeLa}$ cell lysates (Figure 2D and E), thus indicating that both antibodies were able to detect not only exogenous ALADIN but also endogenous ALADIN of molecular weight $60 \mathrm{kDa}$.

To investigate the expression of ALADIN in human tissues, western blot analysis was performed using antibody CNE19. As tissue availability was limited, we analysed ALADIN in human adrenal gland, pancreas, pituitary, kidney, skeletal muscle and placenta samples. Although several non-spe- 


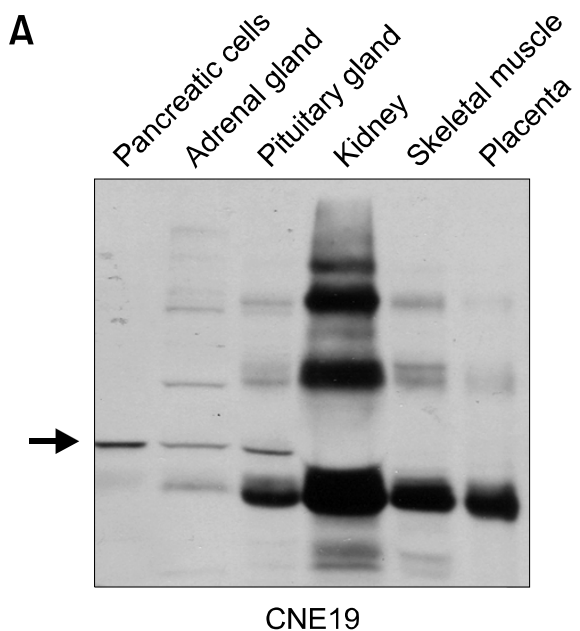

CNE19

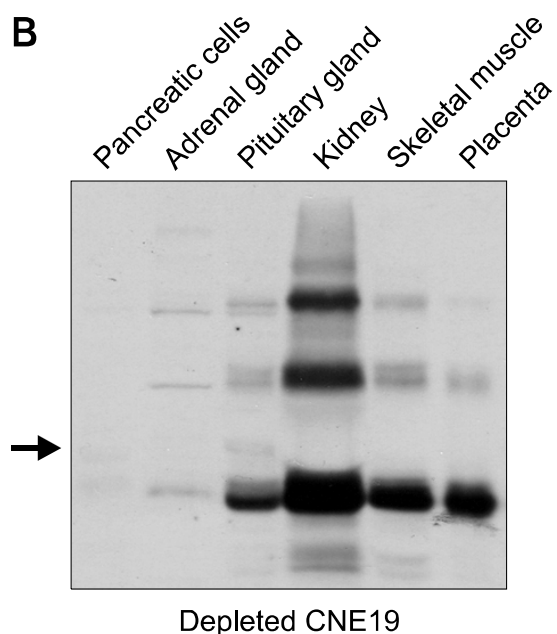

Depleted CNE19
Figure 3. Expression of ALADIN in human tissues. Fifteen $\mu \mathrm{g}$ of protein from tissues and primary cells lysates were separated on SDSPAGE. (A) Western blot analysis using anti-CNE19 antibody. The arrow indicates the expression of ALADIN in pancreatic cells, adrenal and pituitary gland cells. (B) Western blot analysis using adsorbed CNE19 antibody. The arrow shows where ALADIN bands (now absent) would be expected.
A

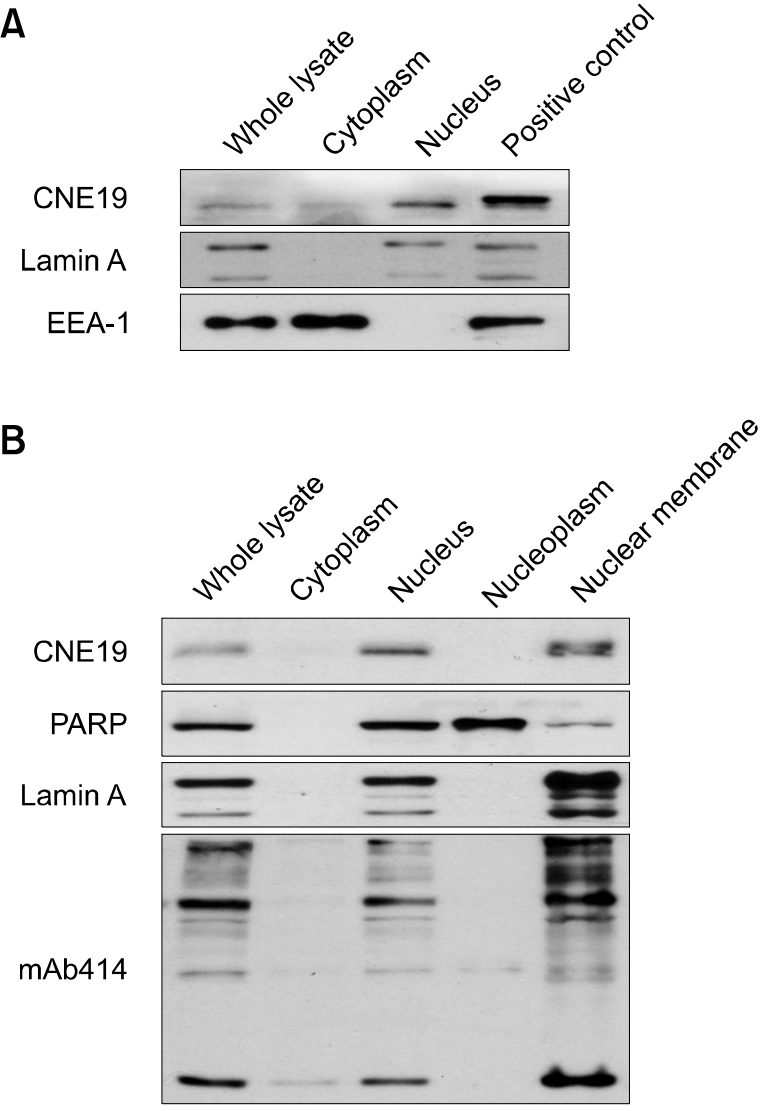

Figure 4. Subcellular localization of ALADIN. (A) HeLa cell lysates were fractionated, and proteins were separated by SDS-PAGE. ALADIN was detected by CNE19 antibody. (B) ALADIN was co-fractionated with the nucleus and the nuclear membrane. Markers for subcellular fractions were EEA-1 (a peripheral membrane protein present in cytosol), PARP (a nuclear protein), Lamin A (a nuclear membrane protein) and mAb414 (NPC, nuclear pore complex). cific signals were present, ALADIN expression was evident in pancreas, adrenal and pituitary gland (Figure $3 \mathrm{~A}$ ). When western blot analysis was carried out with ALADIN-adsorbed CNE19 antiserum, the ALADIN signal was specifically abolished (Figure 3B), while non-specific signals were unaffected. This result clearly indicated that ALADIN was expressed in the tissues mentioned.

Tissue-specific expression of ALADIN raises an interesting point with respect to ALADIN function in cells and focuses attention on tissue-specific symptoms of AS. Our results may suggest that tissuespecific expression of ALADIN in pancreas, adrenal and pituitary gland may reflect the disease-specific phenotype in AS. Interestingly, ALADIN was not detected in kidney, skeletal muscle and placenta, although AAAS mRNA expression was present. Further analysis is required to elucidate the tissuespecific symptoms of Triple A syndrome.

\section{Subcellular localization of endogenous ALADIN}

Previous studies have shown that ALADIN localizes to the NPC using GFP-tagged fusion protein. Therefore, we analysed the subcellular location of endogenous ALADIN using the CNE19 antibody. We observed that ALADIN was present mainly in the nuclear fraction (Figure 4A) as reported previously (Cronshaw and Matunis, 2003). Moreover, ALADIN co-purified with the nuclear membrane when HeLa cells were fractionated into cytoplasm, nucleus, nucleoplasm and nuclear membrane (Figure 4B). Therefore, our data using ALADIN antibody show that endogenous ALADIN is localized in the nuclear membrane, presumably to NPC. 

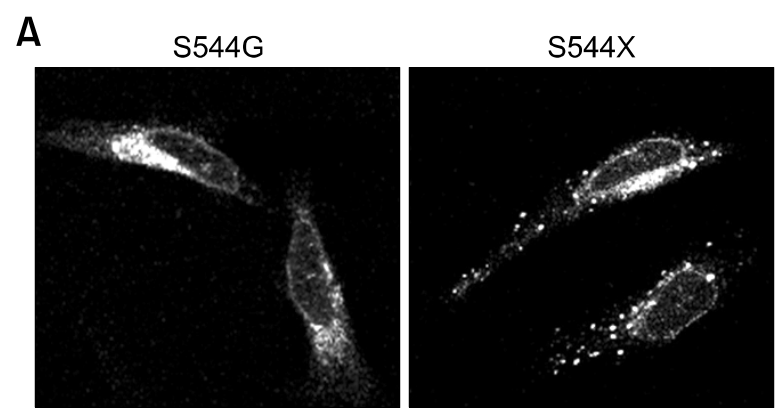

B

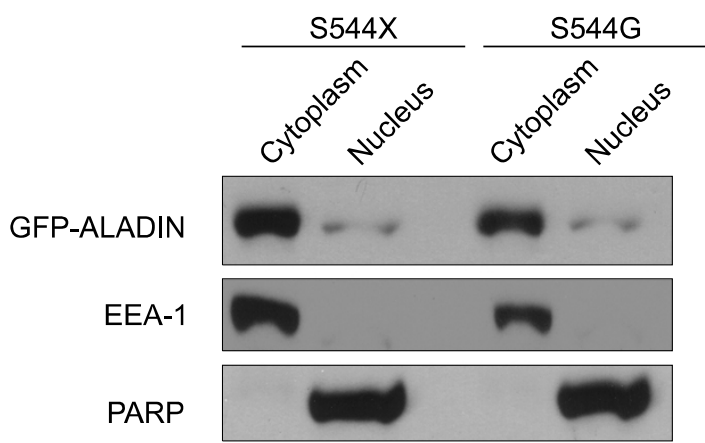

Figure 5. Cellular localization of the mutant ALADIN in HeLa cells. (A) The S544G and S544X mutants were localized in the cytoplasm. (B) Cellular fractionation showed the mutants mainly localized to the cytoplasm.

\section{Subcellular mislocalization of ALADIN mutants}

The C-terminal end of ALADIN contains Ser-HisLeu (Handschug et al., 2001), a putative peroxisomal targeting signal 1 (PTS1) (Gould et al., 1988). Therefore, we examined whether the SHL residues affected correct NPC targeting of ALADIN using artificial mutant constructs S544G and S544X. In contrast to the wt GFP-ALADIN which was localized to the nuclear membrane (Supplemental Data Figure S3), the S544G and S544X mutants were mislocalized to the cytoplasm and formed aggregates (Figure 5A). Cellular fractionation confirmed the cytoplasmic localization of mutants (Figure 5B). These results clearly indicate that the SHL is important for proper NPC targeting. There are several possible causes of mistargeting of these mutants. One possibility is the loss of NPC signal by the partial degradation of the mutant ALADIN. But it does not seem to be the case, since we can detect the same molecular weight mutant GFP-ALADINs as the wt GFP-ALADIN (Supplemental Data Figure S2). Another possibility is that $\mathrm{C}$-terminal region around $\mathrm{SHL}$ is involved in ALADIN's targeting by undefined mechanisms. Those mechanisms may include binding with interacting proteins or its proper folding. Further study is needed to test this possibility. Based on the current understanding of NPC targeting signal of ALADIN, the serine residue at 544 plays a role in targeting of the nascent ALADIN.

\section{Methods}

\section{Cloning of full-length $A A A S$, site-directed mutagenesis and construction of expression plasmids}

The clone, 282D10, was obtained from 1NIB library (Soares et al., 1994) by screening the arrayed library filters with EST 1190E probe using Southern blot analysis. The clone contained $1.4 \mathrm{~Kb}$ insert and the sequence analysis revealed that it contained a partial ORF and a polyadenylation signal. To extend the cDNA, the N-terminus was amplified by 5' RACE methodology using the antisense primer 5'CTTCGCTCCTGAGAGACAGATGG3' and total liver RNA as a template. The full length cDNA of $A A A S$ gene was subcloned at $\mathrm{Xba}$ I site of Flag tagged pYR31 (Min et al., 2003). In addition, the AAAS gene was subcloned at EcoR I and Not I sites of pEGFP-C1.

To generate the mutant constructs, the site-directed mutagenesis was performed using the QuickChange mutagenesis kit (Stratagene, La Jolla, CA) according to the manufacturer's protocol. The primers for the mutant constructs were as follow: GFP-S544G; 5'TCCCCACATGGCCACCTTCCT3', GFP-S544X; 5'ATAGTTTAGCGGCCGCTAATGTGGGGAGTGGGGCA 3'.

\section{Northern blot analysis}

The expression level of $A A A S$ mRNA was analyzed using human multiple tissue northern (MTN) blots according to the manufacturer's instructions (Clontech, Palo Alto, CA). The probes were prepared using Random Primed DNA Labeling Kit (Roche). The 85 bp probe for the first exon was amplified with the following primers, 5' AGATGTGCTCTCTGGGGTTG $3^{\prime}$ and 5' CTCTCATAGCTACTGCCCGT 3'.

\section{Anti-ALADIN antibodies and antibody purification}

Polyclonal antibodies against human ALADIN were raised by immunization of rabbits with synthetic peptides, NELVTGSSYESPPPDFRG (CNE19, aa 23-40) and VLMKGKPRVQDGKPV (CVL16, aa 413-427), both conjugated with keyhole lympet hemocyanin. The final antisera were affinity-purified using the peptides coupled to CNBrSepharose. Depleted CNE19 antiserum was prepared by incubating purified antiserum with bacterially expressed protein extracts containing N-terminal 33 aa of ALADIN.

\section{Western blot analysis}

Human tissues and primary cells used in experiment were obtained from St. Mary hospital of the Catholic university. The western blot analysis was performed as described elsewhere (Harlow et al., 1999). The primary polyclonal 
rabbit antibodies were anti-ALADIN and anti-Lamin A (Cell Signalling Technology, Inc.). The primary mouse monoclonal antibodies were anti-FLAG (Sigma, St. Louis, MO), anti-GFP (Santa Cruz Biotechnology, Inc.), anti-EEA1 (BD Biosciences, San Jose, CA), anti-mAb414 (BAbCO, Richmond, CA) and anti-PARP (BD Biosciences, San Jose, CA). Anti-ALADIN antibodies were used in 1:3000. The detection was achieved using western blotting luminal reagent (Santa Cruz Biotechnology, Inc.).

\section{Cell culture and subcellular fractionation of ALADIN}

HeLa cells were cultured in DMEM supplemented with $10 \%$ calf serum and $50 \mathrm{U} / \mathrm{ml}$ of penicillin/streptomycin. For nuclear and cytoplasmic fractions, cells were lysed in buffer $(10 \mathrm{mM} \mathrm{NaCl}, 1.5 \mathrm{mM} \mathrm{MgCl}, 10 \mathrm{mM}$ Tris- $\mathrm{HCl}$ $(\mathrm{pH} 7.4))$ for $10 \mathrm{~min}$ on ice. Nuclei were pelleted by centrifugation at $1,000 \times g$ for $3 \mathrm{~min}$. The pelleted nuclei were washed twice with lysis buffer. For separation of nuclear membrane, nuclear pellet was lysed in buffer $(20$ $\mathrm{mM}$ HEPES-KOH, $1.5 \mathrm{mM} \mathrm{MgCl} 2,420 \mathrm{mM} \mathrm{KCl}, 0.2 \mathrm{mM}$ EDTA, $25 \%$ glycerol). Nuclear membrane was pelleted by centrifugation at $13,000 \mathrm{rpm}$ for $3 \mathrm{~min}$. Cellular fractionation of mutants was performed using the NE-PER Nuclear and Cytoplasmic Extraction Reagents (Pierce) following the manufacturer's instructions.

\section{Transfection and immunofluorescence}

HeLa cells were plated in 6 -well plates $\left(2 \times 10^{5}\right.$ cells/well $)$ and transfected with $1 \mu \mathrm{g}$ of plasmid DNA using $6 \mu \mathrm{l}(1$ $\mathrm{mg} / \mathrm{ml}$ ) of polyethylenimine (Sigma, St. Louis, MO). Cells grown on cover slips were fixed in $4 \%$ paraformaldehyde and permeabilized with $0.1 \%$ Triton $\mathrm{X}-100$. The cells were stained with anti-mAb414 antibody (BAbCO, Richmond, $\mathrm{CA}$ ) and secondary anti-mouse antibody conjugated to Cy3 (Jackson Laboratories) as manufacturers' recommendation. The signal was observed using Confocal microscope (Bio-Rad).

\section{Supplemental data}

Supplemental Data include three figures and can be found with this article online at http://e-emm.or.kr/article/article files/S P-41-6-02.pdf.

\section{Acknowledgements}

This study was supported by the fund granted from Molecular Medicine Research Group Program (M1-0106-000098) from the Ministry of Science and Technology of Korea.

\section{References}

Brooks BP, Kleta R, Stuart C, Tuchman M, Jeong A, Stergiopoulos SG, Bei T, Bjornson B, Russell L, Chanoine JP, Tsagarakis S, Kalsner L, Stratakis C. Genotypic heterogeneity and clinical phenotype in triple A syndrome: a review of the NIH experience 2000-2005. Clin Genet 2005;68: 215-21

Cronshaw JM, Matunis MJ. The nuclear pore complex protein ALADIN is mislocalizated in triple A syndrome. Proc Natl Acad Sci USA 2003;100:5823-7

Gould SJ, Keller GA, Subramani S. Identification of peroxisomal targeting signals located at the carboxy terminus of four peroxisomal proteins. J Cell Biol 1988;107:897-905

Handschug K, Sperling S, Yoon SJ, Hennig S, Clark AJ, Huebner A. Triple A syndrome is caused by mutations in $A A A S$, a new WD-repeat protein gene. Hum Mol Genet 2001;10:283-90

Harlow E, Lane D. Using Antibodys: A Laboratory Manual 1999;270-304. Cold Spring Harbor Laboratory Press, NY

Li X Ji C, Gu J, Xu J, Jin Z, Sun L, Zou X, Lin Y, Sun R, Wang $\mathrm{P}, \mathrm{Gu} \mathrm{S}$, Mao Y. Molecular cloning and characterization of AAAS-V2, a novel splice variant of human AAAS. Mol Biol Rep 2005;32:127-31

Min KW, Hwang JW, Lee JS, Park Y, Tamura TA, Yoon JB. TIP120A associates with cullins and modulates ubiquitin ligase activity. J Biol Chem 2003;278:15905-10

Moore PS, Couch RM, Perry YS, Shuckett EP, Winter JS. Allgorve syndrome; an autosomal recessive syndrome of ACTH insensitivity, achalasia and alacrima. Clin Endocrinol 1991;34:107-14

Otera H, Okumoto K, Tateishi K, Ikoma Y, Matsuda E, Nishimura M, Tsukamoto T, Osumi T, Ohashi K, Higuchi O, Fujiki Y. Peroxisome targeting signal type 1 (PTS1) receptor is involved in import of both PTS1 and PTS2: Studies with PEX5-defective CHO cell mutants. Mol Cell Biol 1998;18: 388-99

Soares MB, Bonaldo MF, Jelene P, Su L, Lawton L, Efstratiadis A. Construction and characterization of a normalized cDNA library. Proc Natl Acad Sci USA 1994;91: 9228-32

Tullio-Pelet A, Salomon R, Hadj-Rabia S, Mugnier C, de Laet $\mathrm{MH}$, Chaouachi B, Bakiri F, Brottier P, Cattolico L, Penet C, Begeot M, Naville D, Nicolino M, Chaussain JL, Weissenbach J, Munnich A, Lyonnet S. Mutant WD-repeat protein in triple-A syndrome. Nat Genet 2000;26:332-5

Weber A, Wienker TF, Jung M, Easton D, Dean HJ, Heinrichs $C$, Reis A, Clark AJ. Linkage of the gene for the triple A syndrome to chromosome 12q13 near the type II keratin gene cluster. Hum Mol Genet 1996;5:2061-6 